\title{
Experimental study of phase change materials for thermal storage in the temperature range of $300-400^{\circ} \mathrm{C}$
}

\author{
R. Adinberg a and D. Zvegilsky \\ Weizmann Institute of Science, Solar Energy Research Facilities, 76100 Rehovot, Israel
}

\begin{abstract}
Phase change materials (PCM) based on inorganic salts having a temperature of fusion between 300 and $400^{\circ} \mathrm{C}$, were investigated using a lab scale set-up dedicated for studying latent heat storage for concentrating solar thermal power (CSP) technology. This experimental system provides thermal measurements of PCM specimens of about $1000 \mathrm{~g}$ under the heating temperature up to $450^{\circ} \mathrm{C}$ and enables simultaneous investigation of calorimetric properties of the loaded materials and heat transfer effects developed in the thermal storage during the charge and discharge phases. The measurement technique comprised temperature and pressure sensors, a control and data acquisition system and a thermal analysis model used to evaluate the experimental data. Results of the thermochemical tests conducted with a thermal storage medium composed of the ternary eutectic mixture of carbonate salts $\left(34.5 \% \mathrm{~K}_{2} \mathrm{CO}_{3}-33.4 \% \mathrm{Na}_{2} \mathrm{CO}_{3}-32.1 \% \mathrm{Li}_{2} \mathrm{CO}_{3}\right)$ and Diphyl (synthetic thermal oil, max working temperature $400^{\circ} \mathrm{C}$ ) used as the heat transfer fluid are presented and discussed in this paper.
\end{abstract}

\section{Introduction}

Solar thermal storage is a primary method used to stabilize power delivery and prolong the daily operating cycle of the concentrating solar power systems for electricity generation $[1,2]$. The aim of this work was to design a thermal storage module capable of providing high-temperature $\left(300-400{ }^{\circ} \mathrm{C}\right)$ heat to a high-pressure (up to 100 bars) steam generator integrated into a parabolic trough solar thermal electric power plant. The research and development activities have included:

a) Selection of a PCM thermal storage medium based on analytical and experimental studies on materials properties and heat transfer conditions,

b) Simulation of the system heat flow cycles for the phase of charging the storage using the solar field HTF and for the phase of utilizing the thermal storage for steam generation, when the solar power is relatively low or not available, as in the nighttime,

c) Performance analysis of the solar power plant including the developed thermal storage under typical solar input and load demand conditions. The method of solar and fossil energy sources hybridization is used to keep the power plant running 24 hours a day in response to continuous base load demand from the grid.

\footnotetext{
${ }^{a}$ Corresponding author: roman. adinberg@weizmann.ac.il
}

This is an Open Access article distributed under the terms of the Creative Commons Attribution License 4.0, which permits unrestricted use, distribution, and reproduction in any medium, provided the original work is properly cited. 
The thermal storage module has been developed based on the reflux heat transfer storage thermal storage (RHTS) concept described in detail elsewhere [3]. This thermal storage method is aimed at enhancing the dispatchability of utility-scale solar thermal electricity power plants typically operated at a temperature close by $400^{\circ} \mathrm{C}$. Major RHTS features are derived from: 1) utilization of phase change (melting) materials (PCM) for latent heat storage and 2) direct contact between the PCM and a heat transfer fluid (HTF) used to carry over the stored thermal energy from PCM to a steam generator by natural convection. The applied heat transfer mechanism is based on the HTF reflux induced by a combined evaporation-condensation process similar to that used in thermosyphon systems.

One of the key factors limiting the choice of PCM for the RHTS technology, besides the melting point, is its chemical compatibility with the available HTF - the eutectic mixture of biphenyl and diphenyl ether, briefly, Diphyl. This HTF is particular suitable for operational temperatures up to $400{ }^{\circ} \mathrm{C}$ in both liquid and vapour phases, being commercially available as the synthetic thermal oil used in parabolic trough solar power plants [4].

The two options of PCM considered in this study were:

1. Potassium nitrate $\mathrm{KNO}_{3}$ (melting point $334{ }^{\circ} \mathrm{C}$, latent heat of fusion $110 \mathrm{~J} / \mathrm{g}$, thermal conductivity $0.4 \mathrm{~W} / \mathrm{m}-\mathrm{K}$ ) that was extensively studied in the laboratory and found adequately compatible in the solid and molten states with Diphyl up to a temperature of $360^{\circ} \mathrm{C}$ [5].

2. An alternative PCM being investigated in the lab is the ternary eutectic mixture of carbonate salts: $34.5 \% \mathrm{~K}_{2} \mathrm{CO}_{3}, 33.4 \% \mathrm{Na}_{2} \mathrm{CO}_{3}$ and $32.1 \% \mathrm{Li}_{2} \mathrm{CO}_{3}$, called below as $3 \mathrm{C}$ (melting point from $392-397^{\circ} \mathrm{C}$, latent heat about $275 \mathrm{~J} / \mathrm{g}$, thermal conductivity $2.0 \mathrm{~W} / \mathrm{m}-\mathrm{K}$ [6]). This substance is preferable for thermal storage as compared to $\mathrm{KNO}_{3}$ in terms of the higher phase change temperature and the significantly greater energy density and thermal conductivity. This paper presents the results of an experimental study conducted with the $3 \mathrm{C} \mathrm{PCM}$ for thermal storage approaching a temperature of $400^{\circ} \mathrm{C}$.

\section{Experimental studies}

\subsection{Thermogravimetry of 3C PCM}

The ternary carbonate salt 3C was prepared from chemicals of analytical purity. Samples of a few mg were subjected to thermogravimetric measurements by using a TG analyzer SDT Q600 in the temperature range of $350-450^{\circ} \mathrm{C}$. Testing procedure included a number of consecutive heating-cooling cycles at a temperature ramp of $10^{\circ} \mathrm{C} / \mathrm{min}$ in order to determine the temperature and heat of phase change both for melting and solidification processes.

The results of measurements shown in Fig. 1 yielded a $3 \mathrm{C}$ melting temperature ranged between 392 and $394{ }^{\circ} \mathrm{C}$ and the heat of fusion of about $280 \mathrm{~J} / \mathrm{g}$. These values are quite consistent with the thermodynamic data available in the literature [6]. In comparison to nitrate salt $\mathrm{KNO}_{3}$ the $3 \mathrm{C} \mathrm{PCM}$ has a higher phase change temperature and 2-3 times greater energy density that should significantly promote the efficiency of thermal storage.

\subsection{DSC analysis of the 3C-Diphyl chemical system}

High pressure crucibles (up to 20 bars and 30 microliter capacity) were used to study chemical compatibility and thermal stability of the PCM-HTF system composed respectively of 3C (12.8 mg) and Diphyl $(10.8 \mathrm{mg})$ per sample. The experiments were conducted with a DSC 4000 Perkin Elmer instrument by repeating a heating and cooling cycle under comparable conditions (temperature range $350-420^{\circ} \mathrm{C}$ ) to that done in the thermogravimetric measurements with $3 \mathrm{C}$ only (Fig. 1).

It can be seen that the results of Fig. 2 for the 3C-Diphyl system are almost identical to those presented in Fig. 1 for 3C-only in terms of the number of peaks, their shapes and positions on the 


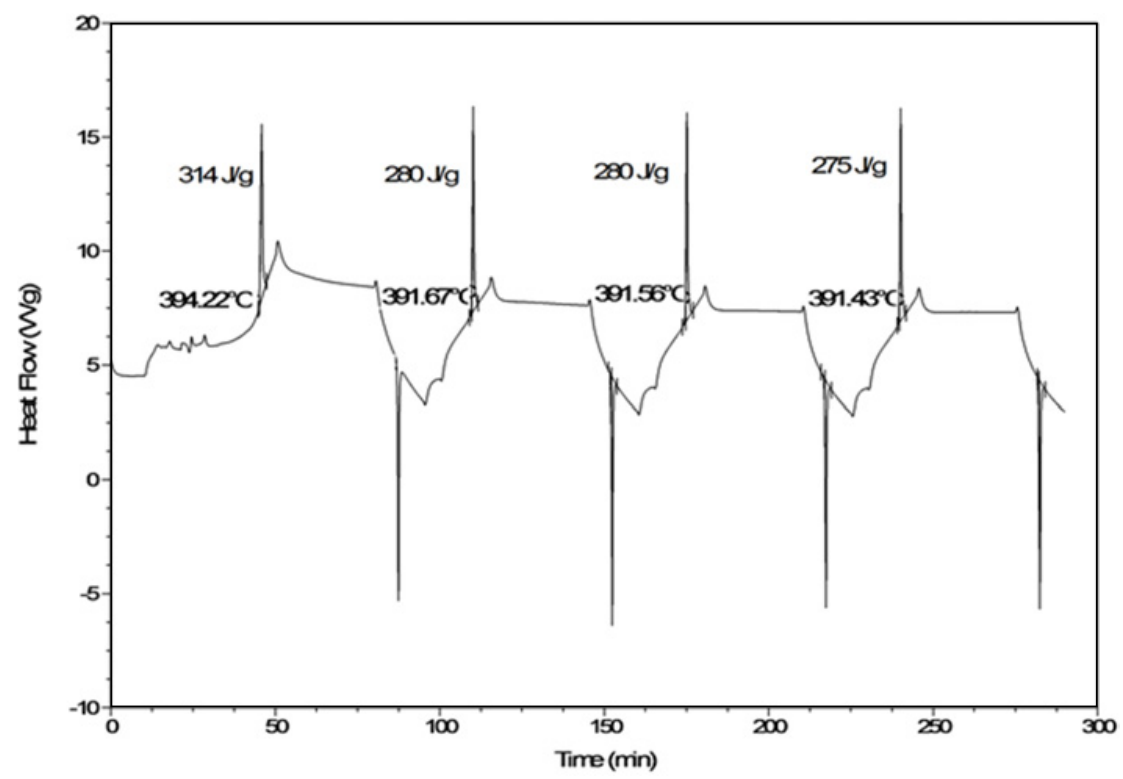

Figure 1. Thermogravimetric measurements with 3C PCM through 4 sequential heating-cooling cycles.

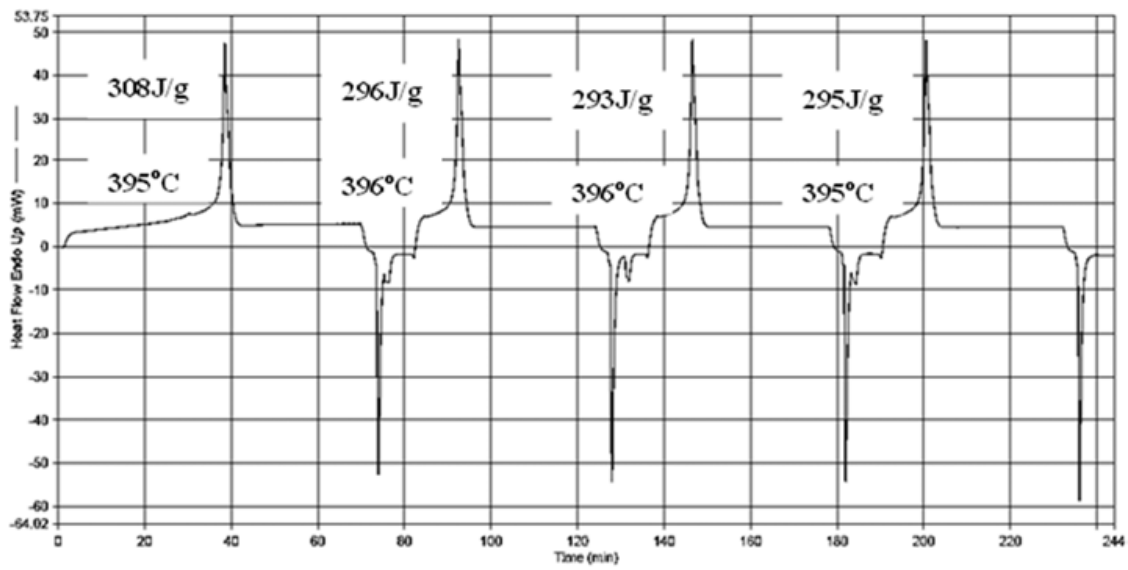

Figure 2. Calorimetric results for the 3C-Diphyl chemical system including 4 sequential heating-cooling cycles ranged from 350 to $420^{\circ} \mathrm{C}$.

temperature scale. Peaks indicate the melting/solidification temperatures and an integral over a heat flow peak gives the heat of phase change. Note that somewhat higher values of phase change heat in Fig. 2 as compared to Fig. 1 are due to the presence of Diphyl in the chemical system.

Since Fig. 2 shows no additional peaks in comparison to Fig. 1 that could be associated with absorption or release of heat due to chemical reactions between $3 \mathrm{C}$ and Diphyl, this fact encourages further study of $3 \mathrm{C}$ as the potential thermal storage PCM for the RHTS method.

\subsection{Mini-reactors}

Long-term tests with the 3C-Diphyl chemical system were carried out using two mini-reactors shown in Fig. 3 for several months. The reactors were bonded together and placed at the center of a furnace to be 


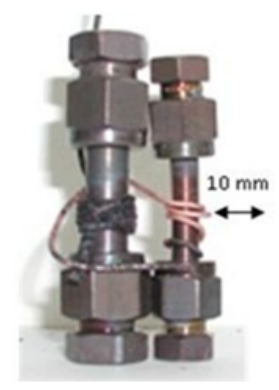

Figure 3. Mini-reactors for testing 3C-Diphyl stability.

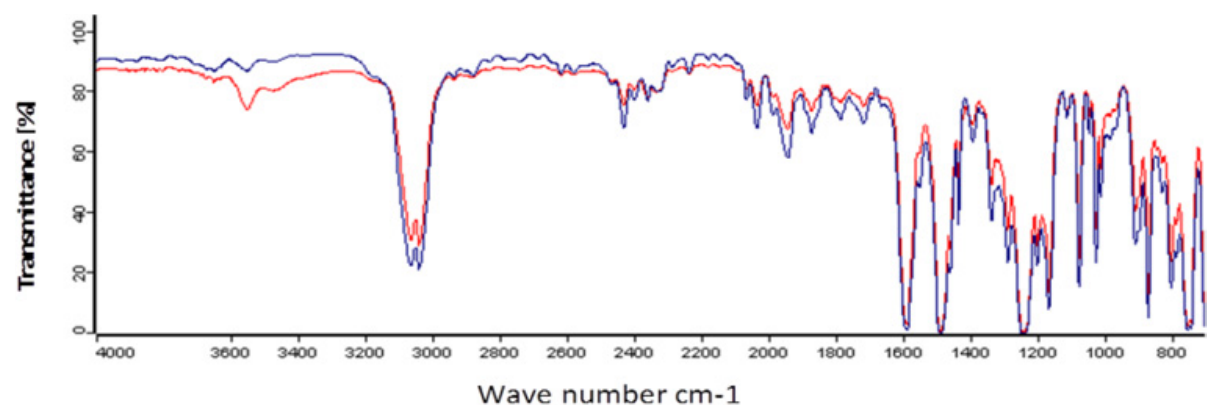

Figure 4. IR spectrums of Diphyl after completing the tests with the mini-reactors. Blue line - the Diphyl-onlyreactor; Red line - the 3C-Diphyl-reactor.

equally heated in experiments. The left hand side reactor contained the $3 \mathrm{C}$ (1g) - Diphyl (1g) chemicals, the other - Diphyl only ( $\mathrm{g}$ ). These reactors were weighed before and after the experiment to ensure no leaking through the seals. In each run, the reactors were maintained at a temperature of $400{ }^{\circ} \mathrm{C}$ for 8 hours then cooled to the room temperature.

The heating-cooling cycles were repeated continuously, however, after every several cycles both reactors were opened to take drop samples of Diphyl for running them through a FTIR spectrometer TENSOR-27 for comparison with the spectrum of fresh Diphyl. Then, the reactors were sealed again to proceed with the experiment. Figure 4 shows the Diphyl spectrums sampled from both reactors at completing the long-term tests. Practically, no difference seen between the two spectrums exhibits an adequate stability of the 3C-Diphyl chemical system within the tested temperatures up to $400{ }^{\circ} \mathrm{C}$.

\subsection{Lab-scale set-up}

A further experimental study of the 3C-Diphyl chemical system was done using a lab scale set-up shown in Fig. 5 and described in detail elsewhere [3]. The amounts of 3C and Diphyl loaded into the sampler vessel were $900 \mathrm{~g}$ and $250 \mathrm{ml}$, respectively. For heating the sample vessel, the temperature controlled furnace was set at $405^{\circ} \mathrm{C}$ to be $10^{\circ} \mathrm{C}$ over the $3 \mathrm{C}$ melting point and the metering valve was closed to prevent heat transfer from the storage due to HTF convection.

After reaching the required temperature, the furnace was switched off and the metering valve was opened to allow HTF to flow and circulate in the closed loop formed by the sample vessel together with vertical pipes (1) and (2) and coil (3) located in the water filled condenser.

During the period of discharge, the heat of the storage is transferred to the water condenser by HTF vapors due to the subsequent processes of: a) evaporation of the liquid Diphyl taking place in the 


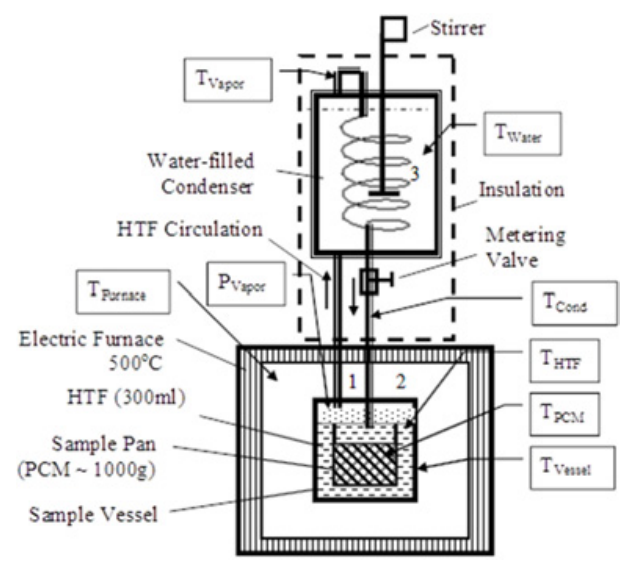

Figure 5. Layout of the lab set-up with the sample vessel containing PCM and HTF. Points of measurements are: $\mathrm{P}$ - pressure, $\mathrm{T}$ - temperature.

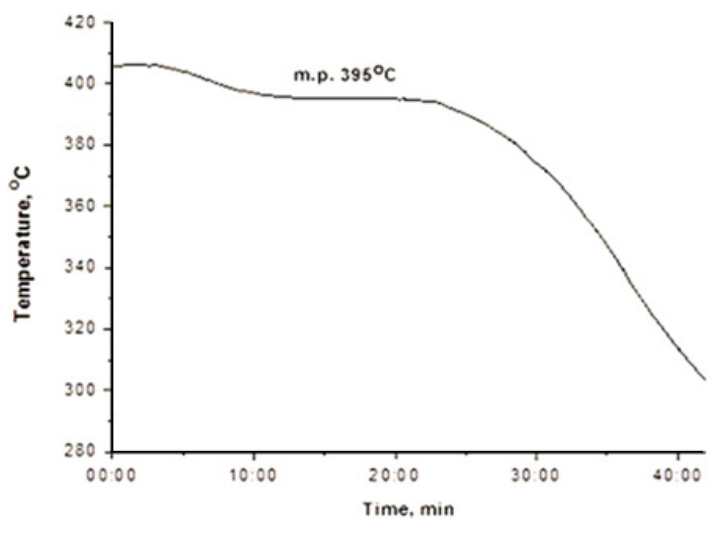

Figure 6. Typical $3 \mathrm{C}$ heat discharge temperature profile measured in the sampler vessel.

sample vessel, b) rising of the vapors in the pipe (1) and c) vapor condensation occurring in the coil (3). By gravity the HTF condensate returns through the pipe (2) back to the sample vessel. The points of measurements include temperature in different locations of the system and HTF vapor pressure, as shown in Fig. 5.

Multiple cycles with different rates of heat charge and discharge were performed by controlling the furnace and metering valve in the desired way. From the thermal energy balance, the heat gained by the water is equal to the decrease of enthalpy of the PCM sample, taking into account the contributions of the receptacle and heat losses that were estimated using the thermal analysis model described in [5].

Figure 6 presents temperatures measured inside the $3 \mathrm{C}$ bulk in the sampler vessel during a heat discharge cycle. It explicitly shows a temperature plateau at the freezing point that is characteristic for molten salt solidification during heat release. The appearance of 3C PCM following a few months of experiments is shown in Fig. 7. From examining the 3C samples on TGA, no changes were found for the specified properties of melting point and heat of fusion as compared to the thermodynamic data [6]. Another evidence for the proper operation of the 3C PCM was a FTIR spectrum of the processed Diphyl that revealed no difference with the spectrum obtained for fresh Diphyl, very similarly to that illustrated in Fig. 4. 


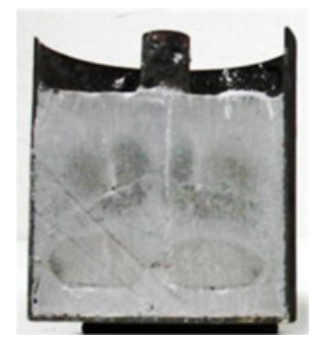

Figure 7. Cross section of the sample vessel containing 3C PCM (HTF removed) after the long-term test.

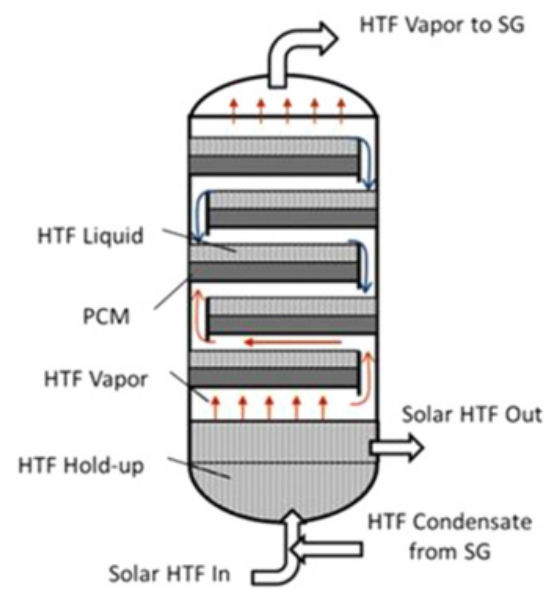

Figure 8. Schematic design of a thermal storage tank, where Diphyl is used as HTF in both the storage and the solar collector.

The effective thermal conductivity coefficient representing the rate of heat transfer by natural convection between the sample vessel (i.e. thermal storage) and the condenser (i.e. heat sink) that was obtained from the thermal analysis model for the heat discharge process is quite high, about $5 \mathrm{~kW} / \mathrm{m}-\mathrm{K}$. This value is comparable to that reported for the $\mathrm{KNO}_{3} \mathrm{PCM}$ in [5] and it is associated with some other thermal applications utilizing reflux condensation heat transfer, such as thermosyphons and pool boilers [7].

\section{Thermal storage tank}

Figure 8 shows the RHTS conception of a thermal storage tank, which is the basic part of a thermal storage module, where thermal cycles of heat charge and discharge are carried out. By definition, the heat storage medium comprises some quantities of PCM (molten salt of either potassium nitrate or the ternary carbonate 3C) and HTF (Diphyl) that are proportionally filled into a number of top-open sections arranged along the vertical axis of the storage tank. In each section, the PCM and HTF substances are naturally separated into two layers due to the significant difference in their density: PCM $\sim 1.9-2.5$ and $\mathrm{HTF}<1 \mathrm{~g} / \mathrm{cm}^{3}$ in the whole range of working temperatures. The storage sections are spaced so that some part of the tank is set free of the storage materials to allow natural convection of HTF vapours rising up and the condensed liquid streaming down.

The HTF pool located at the bottom of the tank is used to charge the thermal storage. It can be directly connected to the solar-heated HTF line, provided the same HTF (Diphyl) is used in both 
Table 1. Calculated parameters of a thermal storage tank.

\begin{tabular}{|l|c|c|}
\hline PCM & $\mathbf{K N O}_{\mathbf{3}}$ & $\mathbf{3 C}$ \\
\hline Amount PCM, ton & 17,000 & 14,000 \\
\hline Amount HTF, ton & ca. 2,000 & ca. 1,000 \\
\hline Height, $\mathrm{m}$ & 20 & 20 \\
\hline Diameter, $\mathrm{m}$ & 32 & 24 \\
\hline Thermal capacity, $\mathrm{MW}_{\text {th }} \mathrm{h}$ & 500 & 1000 \\
\hline
\end{tabular}

systems, but a heat exchanger is to be applied if the HTF chemicals are different. During heat charge cycles, the HTF pool boils continuously producing an intensive flow of vapors reaching the storage sections. By condensing on the relatively cold surface of PCM, the latent energy of vapors is transferred across the PCM layer to heat up and melt the salt. The condensate is partially held off above the PCM layers in the storage sections, while the rest is collected at the bottom. The boiling of the HTF pool is maintained as long as there is solid PCM, which serves as a heat sink. On discharge, the heat flow direction is reversed. Molten salt heats up the liquid HTF causing the vapors to flow up to the top exit toward the steam generator (SG), where the heat of HTF is utilized to generate steam by condensation on the SG heat transfer surface that separates the HTF and water/steam mediums.

The estimates of mass and heat balances for a thermal storage tank working either on potassium nitrate or 3C-PCM are shown in Table 1. For a 50 MWe solar power plant, a thermal storage module having a thermal capacity of 15 full load operational hours should comprise 4 storage tanks with potassium nitrate or 2 storage tanks with $3 \mathrm{C}$ PCM. The amount of HTF is given as an introductory value at this time and will be more accurately determined from heat transfer simulations for the thermal storage sections.

\section{Conclusions}

The results of testing the 3C-Diphyl composition for thermal storage using chemical reactors of various mass scales, from a few $\mathrm{mg}$ in the DSC analysis to $900 \mathrm{~g}$ of the PCM in the lab set-up, were clearly positive in terms of chemical stability of the chemicals up to a temperature of $400{ }^{\circ} \mathrm{C}$. The maximum temperature of Diphyl stability in the thermal storage system tested was found practically the same as that claimed by manufacturers of the Diphyl-based commercial heat transfer fluids used in parabolic trough solar power plants. These properties make the storage medium 3C-Diphyl suitable for the heat transfer efficient method of storing thermal energy (RHTS) that integrates the PCM solid-liquid and HTF liquid-vapour reversible phase change processes, both running simultaneously for respectively storing and transferring heat in the thermal storage system.

This research was sponsored by the Ministry of Energy and Water Resources of Israel according to Contract No. 210-11-022/2010-1-62.

\section{References}

[1] U. Herrmann, D.W. Kearney, Transactions of the ASME, J. Sol. Energy Eng., 124, 145 (2002)

[2] R. Adinberg, Applied Thermal Engineering 31, 3588 (2011)

[3] R. Adinberg, D. Zvegilsky, M. Epstein, Energy Conversion and Management, 51, 9 (2010)

[4] Dowtherm A: http://www.dow.com/heattrans/products/synthetic/dowtherm.htm

[5] R. Adinberg, D. Zvegilsky, Proc. of the ASME 2012 Congress (Houston, USA, Nov. 9-15, 2012)

[6] G. J. Janz and M. R. Lorenz, J. Chem. Eng. Data, 6, 321 (1961)

[7] J.G. Collier and S.R. Thome, Convective boiling and condensation (Clarendon Press, Oxford, 3rd edition, 2001) 